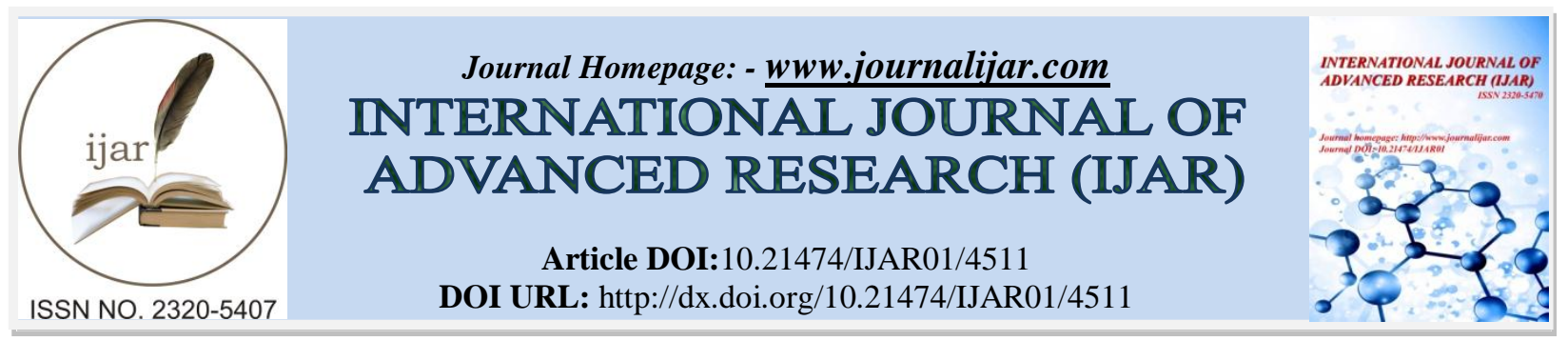

RESEARCH ARTICLE

\title{
COMPARATIVE STUDY OF LED FLUORESCENT MICROSCOPY WITH THE CONVENTIONAL ZN BASED MICROSCOPY FOR DIAGNOSIS OF MICROBIOLOGICALLY CONFIRMED TB CASES.
}

\author{
Pratik Kumar ${ }^{* 1,2}$, Prashant Goswami ${ }^{2}$, Rashmi Kumari ${ }^{2}$, Maneesh Kumar ${ }^{3}$ and Subham Kumar ${ }^{1}$. \\ 1. Department of Biotechnology, College of Commerce, Arts \& Science (Magadh University, Bodh Gaya), Patna, \\ Bihar, India. \\ 2. Intermediate Reference Laboratory, TBDC building, Patna, Bihar, India. \\ 3. Department of Virology, Rajendra Memorial Research Institute of Medical Sciences (ICMR), Agam kuan, Patna, \\ India.
}

\section{Manuscript Info}

Manuscript History

Received: 17 April 2017

Final Accepted: 19 May 2017

Published: June 2017

Key words:-

Ziehl Neelsen, Light Emitting Diode, Auramine, WHO.

\section{Abstract}

Conventional light microscopy of Ziehl Neelsen (ZN) stained smears prepared directly from sputum specimens is the most widely available test for diagnosis of microbiologically confirmed tuberculosis (TB) in India. The $\mathrm{ZN}$ based microscopy needs concentration of $5000-10000$ acid fast bacilli per $\mathrm{ml}$ for its detection and hence reported to detect only $60-70 \%$ TB cases. On the other hand, LED Fluorescent Microscopy (LED FM) with auramine based staining claims to be a faster and more sensitive technique than the conventional microscopy. In the present work, we have evaluated both the techniques taking culture as the reference standard. We have taken sputum samples of 300 presumptive MDR TB patients and tested by all three techniques. 120 samples were positive by $\mathrm{ZN}$ microscopy. Along with these, additional 21 samples were reported positive by LED FM. Out of 300 samples inoculated on culture, 169 samples turned positive. All the culture negative samples were smear negative by both the microscopy techniques. So, we observed that by using LED FM, we can enhance the positivity rate up to $17.5 \%$. We concluded that the LED FM is comparatively more sensitive technique than the conventional light microscopy. Our findings provide support to the World Health Organization (WHO) policy recommendation that conventional light microscopy to be replaced by LED fluorescent microscopy using auramine staining in all settings for clinical sample testing.

Copy Right, IJAR, 2017,. All rights reserved.

\section{Introduction:-}

Tuberculosis (TB) is an infectious disease caused predominantly by Mycobacterium tuberculosis, and transmitted by inhalation of infectious droplet nuclei. Emergence of drug resistant TB is the biggest threat to the global TB control [1-3]. Rapid diagnosis of TB and early treatment initiation is important to control the transmission of infection [4-7]. TB is diagnosed by smear microscopy in many developing countries $[8,9]$. For a long time, diagnosis of TB was dependent on Ziehl Neelsen smear microscopy with the light microscope [10]. The ZN based microscopy needs concentration of $5000-10000$ acid fast bacilli per $\mathrm{ml}$ for its detection [11, 12]. Smear microscopy with the light microscope is a relatively less sensitive method for the diagnosis of TB $[13,14]$ and can only detects $60-70 \%$ of the 
TB cases [15]. An alternative for the light microscope is the fluorescent microscope. In this technique, the fluorescent bacilli of $M$. tuberculosis can be seen at lower magnification and the smears can be examined in less time [16]. However the implementation of this technique was difficult due to higher cost associated with purchase of the microscope with a mercury vapor lamp. Replacement of mercury vapor lamp with a LED illumination system decreases the cost and increases the life span [17]. The LED fluorescent microscopy is known to increase the positivity rate up to $10 \%$ [18]. Culture is still the gold standard for TB diagnostics but its use for routine diagnosis [19] is limited due to the long turnaround time [20]. Need of more rapid and effective diagnostics is essential for effective control of TB [21]. WHO endorsed the LED FM over conventional light microscopy for detection of acid fast bacilli on clinical specimens. In the current research, we have done a comparative study of LED Fluorescent Microscopy with the conventional ZN based microscopy on Presumptive Multi drug resistant (MDR) TB samples to evaluate the need to replace conventional light microscope with LED FM for effective TB control.

\section{Methods and Materials:-}

Intermediate Reference Laboratory (IRL) Patna is certified for various culture and molecular based diagnostics of TB and its drug susceptibility testing with various anti TB drugs. Clinical samples of presumptive MDR TB patients are being received for detection of MDR TB cases. These samples are used for the current study. 300 clinical specimens are taken for the study. Two slides of each sample were prepared and labeled as ' $A$ ' and ' $B$ '. 'A' slides were stained by $\mathrm{ZN}$ staining technique and tested under light microscope, while the 'B' slides were stained by auramine staining technique and observed under LED FM. Also, all the 300 samples were processed and inoculated on solid Lowenstein Jensen (LJ) media for reference standard.

\section{Procedure for ZN staining and Microscopy:-}

Smears were prepared from direct sample. Air dried and heat fixed slides were arranged on staining racks. $1 \%$ filtered carbol fuchsin applied on slides and heated gently. After 5 minutes slides were rinsed with tap water. 25\% sulphuric acid was poured on slides and allowed to stand for 2 to 4 minutes depending on the decolorization of slides. Decolorizer was removed by rinsing with tap water. Finally, $0.1 \%$ methylene blue was applied for 30 seconds and rinsed gently with tap water. Slides were examined under the binocular microscope using 40X lens to select the suitable area and then examined under 100X lens using a drop of immersion oil.

\section{Procedure for auramine staining and Microscopy:-}

Heat fixed slides were flooded with filtered $0.1 \%$ auramine solution and kept for at least $20 \mathrm{~min}$, make sure that the smear area is continuously covered with auramine by adding more if needed. Rinsed with water and drained. $0.5 \%$ acid alcohol was applied for 3 minutes followed by tap water. Flooded smear with $0.5 \%$ potassium permanganate solution for 1 minute. Time is critical because counter staining for longer time may quench the acid fast bacilli. Gently rinsed with water and drained. Air dried on a slide rack away from sunlight. Examined using 20X objective for focusing and $40 \mathrm{X}$ objective for further reading.

\section{Procedure for Culture Inoculation:-}

Equal volume of freshly prepared NALC NaOH decontaminating solution (contains $2 \% \mathrm{NaOH}, 1.45 \%$ trisodium citrate and $0.5 \%$ NALC) was added into the sample and incubate for 15 minutes at room temperature with repeated vortexing to ensure proper liquefication. Phosphate buffer saline (PBS) was added to the $45 \mathrm{ml}$ mark of the falcon tube to neutralize the $\mathrm{pH}$. Centrifuge the samples at $3000 \mathrm{X} \mathrm{g}$ for 20 minutes at $4^{\circ} \mathrm{c}$. After centrifugation, supernatant was discarded and pellet was used for inoculation on Lowenstein Jensen (LJ) media. Inoculated culture tubes were incubated at $37^{\circ} \mathrm{c}$ and observed for growth.

The entire sample handling procedure was done inside Bio safety cabinet (BSC) by certified lab staff. Only after heat fixing of slides, it could be processed outside BSC for staining and microscopy. The staining reagents and sample processing reagents were passed on recommended quality control test and were within their expiry date.

\section{Results:-}

Out of 300 samples tested by all three techniques mentioned above, 120 samples were positive by ZN microscopy. Along with these, additional 21 samples (total 141samples) were reported positive by LED FM. A total of 169 samples turned positive on culture which includes 141 smear positive and additional 28 smear negative samples. All the culture negative samples were smear negative by both the microscopy techniques. 


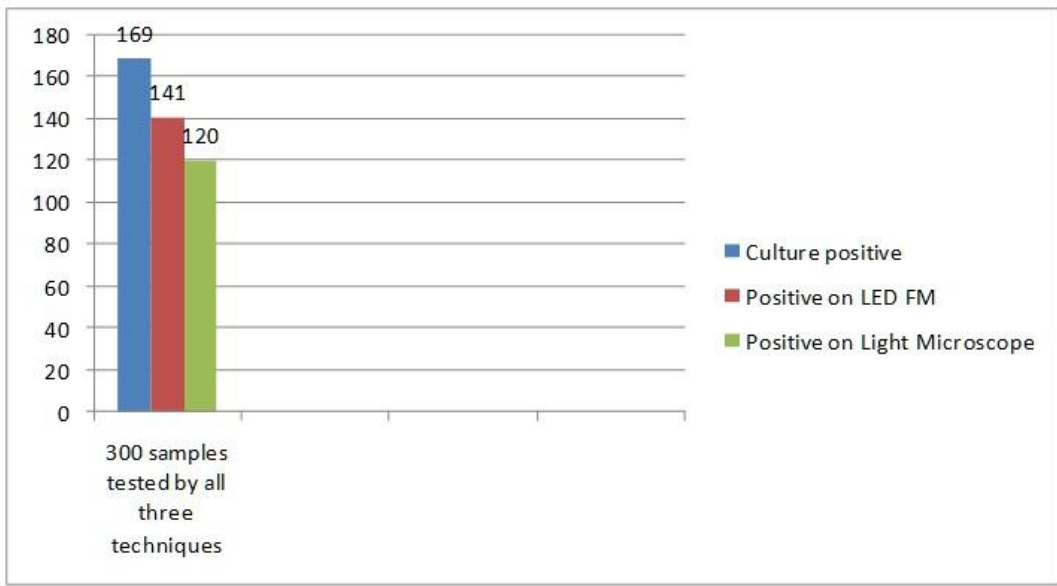

Figure1:- Performance of LED FM and ZN based technique taking culture as reference

\section{Discussion:-}

In this era of molecular and other rapid TB diagnostic techniques, microscopy is still the most utilized technique in India. Under Revised National TB Control Programme (RNTCP), we have one designated microscopy centre (DMC) for every 100,000 population [22]. These DMCs are responsible for detection of all the new TB cases and hence the improvement in case detection is directly related with the improvement in microscopy. Our study showed that LED FM increases the positivity rate by $17.5 \%$. We observed the sensitivity of ZN based microscopy around $71 \%$ and that of LED FM around $83 \%$. We observed $100 \%$ specificity of both the techniques in our study. It was observed that implementation of LED FM services can increase the smear positivity rates at DMC level [23-25]. Similar results have also observed in China [26]. Two reasons may justify this increase in positive results, first is the stronger absorbability of mycolic acid for carbol auramine than carbol fuchsin leading to larger number of acid fast bacilli stained with FM as compared to ZN [27, 28], second is the larger field area examined using high power fields using FM as compared to oil immersion fields by ZN. In addition of this increased positivity rate, there is another advantage of using LED FM is that it takes less time to read the slides than the light microscopy. So implementing this technique at DMC level will improve the performance and efficiency of the lab. We have done our work on samples of presumptive MDR TB patients at IRL level. The significance of using this technique at IRL is that the smear result is critical to decide the diagnostic algorithm for further testing. If a sample is smear positive, further testing will be done by Line probe assay (LPA) which gives the susceptibility of both rifampicin and isoniazid, while the smear negative samples will be tested either by culture or Cartridge based nucleic acid amplification test (CBNAAT). The culture takes long time to give the results and the CBNAAT gives susceptibility of rifampicin only. For a presumptive MDR TB case tested by CBNAAT and resulted as MTBC positive with rifampicin sensitive or resistant, it must be evaluated for isoniazid also by culture and DST for more effective treatment [29]. This isoniazid susceptibility testing by culture takes more time. If we can improve the smear positivity, we can also reduce these cases. So, using LED FM can improve the utilization of LPA for more effective patient management.

\section{Conclusion:-}

Utilization of LED Fluorescent Microscopy in place of light microscopy enhances the positivity rate up to 17.5 $\%$.Our findings provide support to the World Health Organization (WHO) policy recommendation that conventional light microscopy can be replaced by LED fluorescent microscopy using auramine staining in all settings for clinical sample testing.

\section{Acknowledgement:-}

We thank The Director, IRL Patna for the support and motivation. We thank Dr Jainendra Kumar, Professor, Department of Biotechnology, College of Commerce, Arts \& Science for his continuous faith and support. We are also thankful to the Lab team of IRL Patna for their help. 


\section{References:-}

1. Control, C.f.D. and Prevention, Emergence of Mycobacterium tuberculosis with extensive resistance to second-line drugs--worldwide, 2000-2004. MMWR. Morbidity and mortality weekly report, 2006. 55(11): p. 301.

2. Prasad, R., Multidrug and extensively drug-resistant TB (M/XDR-TB): problems and solutions. The Indian journal of tuberculosis, 2010. 57(4): p. 180-191.

3. Gandhi, N.R., et al., Extensively drug-resistant tuberculosis as a cause of death in patients co-infected with tuberculosis and HIV in a rural area of South Africa. The Lancet, 2006. 368(9547): p. 1575-1580.

4. Storla, D.G., S. Yimer, and G.A. Bjune, A systematic review of delay in the diagnosis and treatment of tuberculosis. BMC public health, 2008. 8(1): p. 15.

5. Dheda, K., et al., Early treatment outcomes and HIV status of patients with extensively drug-resistant tuberculosis in South Africa: a retrospective cohort study. The Lancet, 2010. 375(9728): p. 1798-1807.

6. Yimer, S., G. Bjune, and G. Alene, Diagnostic and treatment delay among pulmonary tuberculosis patients in Ethiopia: a cross sectional study. BMC infectious diseases, 2005. 5(1): p. 112.

7. Lienhardt, C., et al., Factors affecting time delay to treatment in a tuberculosis control programme in a sub-Saharan African country: the experience of The Gambia. The International Journal of Tuberculosis and Lung Disease, 2001. 5(3): p. 233-239.

8. Corbett, E.L., et al., The growing burden of tuberculosis: global trends and interactions with the HIV epidemic. Archives of internal medicine, 2003. 163(9): p. 1009-1021.

9. Dye, C., et al., Evolution of tuberculosis control and prospects for reducing tuberculosis incidence, prevalence, and deaths globally. Jama, 2005. 293(22): p. 2767-2775.

10. Steingart, K.R., A. Ramsay, and M. Pai, Optimizing sputum smear microscopy for the diagnosis of pulmonary tuberculosis. Expert review of anti-infective therapy, 2007. 5(3): p. 327-331.

11. Ahmad, K. and F. Khurshid, An Update on the Laboratory Diagnosis of Tuberculosis. Journal of Islamabad Medical \& Dental College (JIMDC), 2015. 4(1): p. 42-47.

12. HASSEN, M.S., PULMONARY TUBERCULOSIS AND HEMATOLOGICAL PROFILES AMONG TUBERCULOSIS SUSPECTED PATIENTS IN SELECTED HOSPITALS OF OROMIA REGIONAL STATE, ETHIOPIA. 2016, AAU.

13. Hepple, P., N. Ford, and R. McNerney, Microscopy compared to culture for the diagnosis of tuberculosis in induced sputum samples: a systematic review [Review article]. The international journal of tuberculosis and lung disease, 2012. 16(5): p. 579-588.

14. Marais, B.J., et al., Use of light-emitting diode fluorescence microscopy to detect acid-fast bacilli in sputum. Clinical Infectious Diseases, 2008. 47(2): p. 203-207.

15. Teran, R. and J.H. de Waard, Recent advances in the laboratory diagnosis of tuberculosis.

16. Steingart, K.R., et al., Fluorescence versus conventional sputum smear microscopy for tuberculosis: a systematic review. The Lancet infectious diseases, 2006. 6(9): p. 570-581.

17. Organization, W.H., Fluorescent light-emitting diode (LED) microscopy for diagnosis of tuberculosis: policy statement. 2011.

18. Kumar, P., et al., Available online www. jsaer. com. Journal of Scientific and Engineering Research, 2017. 4(1): p. 78-80.

19. Reid, M.J. and N.S. Shah, Approaches to tuberculosis screening and diagnosis in people with HIV in resource-limited settings. The Lancet infectious diseases, 2009. 9(3): p. 173-184.

20. Huebner, R.E., R. Good, and J. Tokars, Current practices in mycobacteriology: results of a survey of state public health laboratories. Journal of Clinical Microbiology, 1993. 31(4): p. 771-775.

21. Alvarez-Uria, G., et al., Rapid diagnosis of pulmonary and extrapulmonary tuberculosis in HIV-infected patients. Comparison of LED fluorescent microscopy and the geneXpert MTB/RIF assay in a district hospital in India. Tuberculosis research and treatment, 2012. 2012.

22. Chadha, S.S., et al., Operational challenges in diagnosing multi-drug resistant TB and initiating treatment in Andhra Pradesh, India. PloS one, 2011. 6(11): p. e26659.

23. Reza, L.W., et al., LED-fluorescence microscopy for diagnosis of pulmonary tuberculosis under programmatic conditions in India. PloS one, 2013. 8(10): p. e75566.

24. Ramsay, A., et al., New policies, new technologies: modelling the potential for improved smear microscopy services in Malawi. PLoS One, 2009. 4(11): p. e7760.

25. Ziaee, M., et al., Comparison of the value of two different sputum staining for diagnosis of acid-fast bacilli. Archives of Clinical Infectious Diseases, 2008. 3(2).

26. Xia, H., et al., Multicentre evaluation of Ziehl-Neelsen and light-emitting diode fluorescence microscopy in China. The International Journal of Tuberculosis and Lung Disease, 2013. 17(1): p. 107-112.

27. Richards, O.W., The Staining of Acid-Fast Tubercle Bacteria. Science (Washington), 1941. 93(2408).

28. Gordon, C., A. Van Deun, and R. Lumb, Evaluating the performance of basic fuchsin for the Ziehl-Neelsen stain. The International Journal of Tuberculosis and Lung Disease, 2009. 13(1): p. 130-135.

29. Revised National TB Control Programme, Technical and Operational Guidelines for Tuberculosis Control in India, 2016. 